\title{
Costo-efectividad de furoato de mometasona tópica nasal para el tratamiento de la rinosinusitis aguda en México
}

\author{
Cost-effectiveness of nasal topical mometasone furoate \\ for the treatment of acute rhinosinusitis in Mexico
}

Kely Rely ${ }^{1}$, Guillermo Salinas E², Silvia Martínez V², Carlos Pérez B³ ,Virginia Blandón $^{3}$, Pierre K. Alexandre

Forma de citar: Rely R, Salinas-Escudero G, Martinez-Valverde S, Pérez C, Blandón V, Pierre A. Costo efectividad de furuato de mometasona tópica nasal para el tratamiento de la rinosinusitis aguda en México. Rev Univ Ind Santander Salud. 2017; 49(2): 340-350. DOI: http://dx.doi.org/10.18273/revsal.v49n2-2017007 @c) (1)

\section{RESUMEN}

Introducción: La rinosinusitis aguda (RSA) es frecuente en primer nivel de atención y los pacientes pueden mejorar sin antibióticos. Objetivo: Análisis de costo efectividad de furoato de mometasona spray nasal (FMSN) comparado con amoxicilina en tratamiento de rinosinusitis aguda en México desde punto de vista del Sistema Nacional de Salud. Método: Análisis costo-efectividad comparando FMSN $200 \mathrm{mg}$ dos veces al día y amoxicilina $500 \mathrm{mg}$ tres veces al día. Resultados en términos de eficacia modelados como cambios en sistema de Calificación de Síntomas Mayores (MSS por siglas inglés). MSS consiste en cinco preguntas: rinorrea, goteo retro nasal, congestión, cefalea sinusal y dolor facial. Datos clínicos obtenidos de ensayo clínico aleatorizado. Costos expresados en pesos mexicanos 2016. Se realizó análisis de sensibilidad univariable y multivariables para algunos parámetros utilizados en el modelo. Resultados: Los costos proyectados fueron de $\$ 3,261$ pesos con FMSN y $\$ 3,438$ pesos con amoxicilina. FMSN fue asociado con ahorro de costos por paciente de $\$ 177$ pesos comparado con amoxicilina en un periodo de dos semanas. La tasa incremental de costo-efectividad del FMSN lo ubica como dominante frente a amoxicilina. El análisis de sensibilidad confirma los ahorros globales de costos y la superioridad en términos de la eficacia. Conclusión: En pacientes con rinosinusitis aguda, no complicada, el tratamiento del, FMSN 200 mcg dos veces al día, produjo mejoras significativas de los síntomas en comparación con amoxicilina en pacientes sin infecciones bacterianas y es una alternativa costo efectiva para el Sistema Nacional de Salud Mexicano. Comentario editorial al finalizar el artículo.

Palabras clave: Rinosinusitis aguda, rinosinusitis, furoato de mometasona, costo-efectividad, tratamiento, Mexico.

\footnotetext{
ABSTRACT

Introduction: Acute rhinosinusitis (RSA) is a common cause of consultation on first level of attention and it can be solved without antibiotics use. Objective: Analysis of cost effectiveness of nasal spray mometasone furoate (NSMF)

1. CEAHT. Ciudad de México, México.

2. Hospital Infantil de México Federico Gómez. Ciudad de México, México.

3. Merck Sharp \& Dohme de México. Ciudad de México, México.

4. Johns Hopkins University. Baltimore, Estados Unidos de América.

Correspondencia: Guillermo Salinas-Escudero. Dirección: Márquez 162. Colonia Doctores. Delegación Cuauhtémoc. CP 06720 Ciudad México. Correo electrónico: guillermosalinas@yahoo.com. Teléfono: + (52) 1552289915 ext. 4520
} 
compared to amoxicillin in treatment of acute rhinosinusitis in Mexico from a National Mexican Health System perspective. Methods: Cost-effectiveness analysis comparing NSMF 200 $\mathrm{g}$ twice daily and amoxicillin 500mg three time daily. The effectiveness outcomes of the study were modeled as changes in the Major Symptom Score (MSS). MSS consists of five questions concerning rhinorrhea, post-nasal drip, nasal congestion, sinus headache, and facial pain. Clinical data were obtained from a randomized clinical trial. Cost were expressed in Mexican pesos 2016. Sensitivity analysis was conducted univariable and multivariable for some model parameters. Results: The projected costs were $\$ 3,261$ pesos with NSMF and $\$ 3,438$ pesos with amoxicillin. NSMF was associated with a cost savings per patient of $\$ 177$ pesos versus amoxicillin over a 2 weeks period. The incremental cost-effectiveness ratio for NSMF dominated amoxicillin. The sensitivity analysis confirms overall cost savings and superiority in terms of effectiveness. Conclusion: In patients with non-complicated acute rhinosinusitis NSMF $200 \mu \mathrm{g}$ twice daily produce significantly an improvement in symptoms compared to amoxicillin in patients without bacterial infections and is a cost-effective alternative for the National Health Mexican System. Editorial comment at the end of this article.

Keywords: acute rhinosinusitis, rhinosinusitis, mometasone furoate, cost-effectiveness, treatment, Mexico.

\section{INTRODUCCIÓN}

La rinosinusitis es la infección y/o inflamación que afecta al menos a uno de los senos paranasales y a la nariz ${ }^{1}$. La etiología más frecuente es la infección viral asociada al resfriado común, que se complica con una infección bacteriana en el $0.5-2 \%$ de los episodios ${ }^{2}$.

Las enfermedades respiratorias representan el 55.6\% de los casos de enfermedad en México (23.7 millones de casos incidentes $)^{3}$ y entre el $4.1 \%$ del total de las consultas de primer nivel (4.1 millones) ${ }^{4}$, conjunto de enfermedades que afecta a buena parte de la población y que representa un problema de salud pública, dentro de las IRA se ubica la rinosinusitis aguda.

La rinosinusitis aguda (RSA) es una de las 10 condiciones más frecuentes en el primer nivel de atención ${ }^{5}$; ya que se conoce que las $2 / 3$ partes de los pacientes con este diagnóstico mejoran sin antibióticos ${ }^{6}$. Sin embargo, se prescriben antibióticos contra la sinusitis en $98 \%$ de los $\operatorname{casos}^{7}$, a pesar de que la fisiopatología de dicha enfermedad tiene un papel importante al componente inflamatorio, como la causa de la sintomatología con la que cursan los pacientes ${ }^{8}$.

Por otro lado, el rol de los esteroides intranasales como antiinflamatorios ha conseguido que algunas de estas sales sean consideradas en el tratamiento, así como disminuyen la frecuencia y gravedad de las recurrencias ${ }^{7}$ De alguna manera, el hecho que esteroides como furoato de mometasona spray nasal (FMSN) consigan dichos efectos de manera segura y eficaz ha permitido que se incluyera dentro de su información para prescribir la indicación de RSA, demostrando un incremento en el beneficio en comparación del tratamiento con antibiótico?.

Los corticosteroides, además de aliviar la rinorrea, prurito nasal y estornudos, son eficaces en reducir la congestión nasal y alivian parcialmente el prurito ocular $^{10-13}$. En el estudio pivote de FMSN en RSA se comparó amoxicilina versus placebo, dónde la administración de $200 \mathrm{mcg}$ dos veces al día, mejoró los síntomas más eficazmente que el placebo o la amoxicilina ${ }^{14}$.

El objetivo del uso de antibióticos en la rinosinusitis aguda, es erradicar la infección bacteriana de los senos paranasales, acelerar la resolución de síntomas y mejorar la calidad de vida. Varios estudios aleatorizados, controlados se han realizado para estudiar los efectos de los antibióticos en la rinosinusitis aguda bacteriana, sin embargo, no se han demostrado diferencias entre las diferentes opciones terapéuticas. La justificación para utilizar amoxicilina como la terapia de primera línea para la rinosinusitis bacteriana aguda, es por su seguridad, eficacia, bajo costo y estrecho espectro microbiológico.

La amoxicilina ha demostrado tener mejores tasas de curación y mejoría clínica en comparación con placebo $^{15-16}$. Por otro lado, también existe evidencia de que la rinosinusitis aguda puede ser tratada sin la necesidad de antibióticos, por lo que la reducción en el uso de los mismos es un objetivo deseable.

En México, como en muchos países del mundo, los antibióticos se utilizan incluso para casos en donde no existe evidencia que sea necesaria esta clase terapéutica, causando uso indiscriminado con la consecuente resistencia bacteriana. De esta manera, se desconoce la importancia del manejo del problema inflamatorio que al ser resuelto con FMSN, permite el alivio de síntomas y la consecuente disminución de un problema de salud como es el uso irreflexivo de antibióticos.

Un estudio realizado por Svensson, et $\mathrm{al}^{17}$ en Suecia, demostró la costo efectividad del furoato de 
mometasona nasal en comparación con amoxicilina o placebo desde la perspectiva de vista de la sociedad. El modelo empleado, utilizó las escalas de síntomas mayores en un periodo de 15 días. Se compararon los costos de los efectos medidos en años de vida ajustados por calidad de los tratamientos y estos fueron calculados al dividir el peso de los puntajes de calidad de vida medidos por el EQ-5D y EQ-VAS correspondientes a cada puntaje de síntomas. Los resultados mostraron que los costos se redujeron y los AVAC aumentaron, con lo que se demostró que el tratamiento con furoato de mometasona es un tratamiento no solo costo efectivo, sino que llega a ser costo ahorrador en algunos escenarios.

En México el tratamiento con Amoxicilina está indicado en las Guías de Práctica Clínicas ${ }^{18}$ como primera línea de tratamiento para los pacientes que presenten RSA, situación que resulta cuestionable ya que no se ha probado su costo efectividad y por lo cual en este estudio se pretende evaluar una opción alternativa de manejo, adicionalmente cabe mencionar que en México se desconoce el costo del tratamiento y la carga de la enfermedad de RSA, en este sentido, el estudio tiene la bondad de realizar una estimación de dicho costo en el entorno Mexicano.

El propósito de este estudio fue la realización de un análisis costo-efectividad del FMSN en comparación con la amoxicilina en el tratamiento de la rinosinusitis en el contexto del Sistema Nacional de Salud Mexicano con la finalidad de emplear de forma más eficiente los recursos escasos con los que se cuenta.

\section{MATERIAL Y MÉTODOS}

Se realizó un estudio para evaluar la relación costoefectividad de FMSN en comparación con amoxicilina en el tratamiento de la RSA. La población del estudio incluyó una cohorte hipotética de pacientes con características similares a los del estudio de Meltzer EO y colaboradores, 14 compuesta por pacientes mayores de 12 años de edad con RSA que no presenten síntomas ni signos de infección bacteriana ${ }^{19-20}$. El estudio se hizo desde la perspectiva del Sistema Nacional de Salud (SNS) mexicano. Los costos de los recursos sanitarios utilizados se reportan en dólares americanos 2016 (\$US).

El parámetro principal de valoración de la eficacia fue la proporción de mejoría de los síntomas de la RSA, según el IRC (Índice Compuesto de Síntomas: dolor facial / dolor a la presión / aumento sensibilidad / cefalea sinusal, rinorrea, congestión / obstrucción nasal y goteo post-nasal) durante el periodo de tratamiento de
15 días. Para cada pregunta hay una escala de gravedad de cuatro niveles, que van desde 0 a 3 (ninguno, leve, moderado, grave $)^{20}$.

El diagrana del modelo se presenta en la Figura 1, se basa en un número de supuestos respecto a la historia natural de la RSA y su manejo. Los supuestos claves se resumen a continuación:

1. Al inicio del tratamiento se consideraron las consultas al médico de primer nivel como las habituales relacionadas con la RSA. Se asumió que un paciente sin fracaso acudiría dos veces a la consulta de primer nivel sobre la base del criterio emitido por el estudio de laboratorio y gabinete que recomienda ${ }^{21}$.

2. Se asumió que un paciente con RSA con persistencia de síntomas mayores en caso de ineficacia, acudiría a la consulta del médico del segundo nivel de atención, y en dependencia de su gravedad, podría tener como promedio cuatro consultas, dos estudios de laboratorios y un estudio de radiodiagnóstico.

En el modelo se evaluaron los resultados según la duración media del estudio de Meltzer EO, et al. ${ }^{14}$ que fue de dos semanas. Tal como lo indican las Guías para la Evaluación Económica de Intervenciones Sanitarias $^{22}$, no se utilizó ninguna tasa de descuento, tanto en los costos como en los beneficios sanitarios considerados en el modelo.

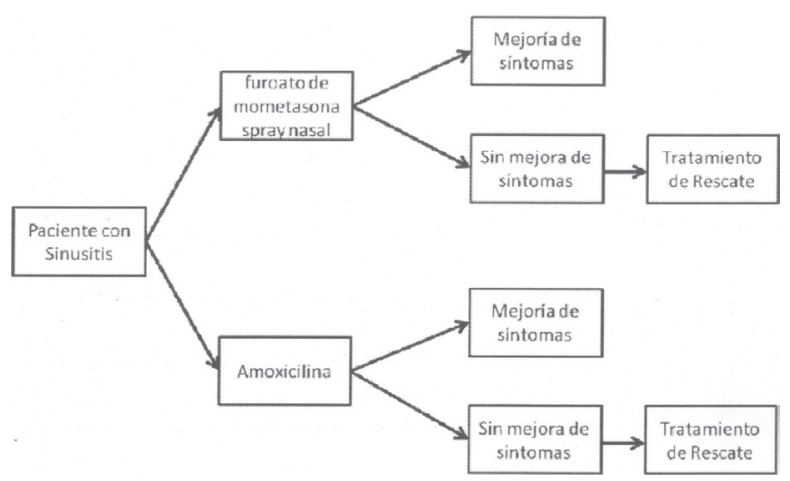

Figura 1. Modelo de Rinosinusitis aguda

Los recursos sanitarios incluidos, y su porcentaje de utilización por parte de los pacientes con RSA, se obtuvieron a través de un panel de expertos con experiencia en el manejo de la RSA, a quienes se les solicitó que respondieran a una encuesta de utilización de servicios sanitarios según los resultados clínicos (fracaso o éxito) descritos en el modelo. Se incluyeron los costos derivados de visitas médicas, estudios de laboratorios y estancias hospitalarias 
A partir de estos datos, se estimó el costo/día por paciente con RSA y se consideró que cada grupo de tratamiento disminuiría este costo medio diario, en la misma proporción en que se reducía la frecuencia de los síntomas. A través de esta encuesta, se estimó el porcentaje de utilización de recursos sanitarios adicionales que se derivan del porcentaje de fracaso por cada alternativa. Los costos totales por pacientes incluyeron los recursos sanitarios por su enfermedad, los recursos sanitarios adicionales debido al fracaso terapéutico y los fármacos.

Los resultados del modelo se expresaron en términos de eficacia y costos diferenciales entre FMSN frente a amoxicilina, así como en términos de razón costoefectividad incremental (RCEI). Se realizó un análisis de sensibilidad univariable y multivariables para algunas de las variables utilizadas en el modelo.

Durante los primeros 14 días, los pacientes en los que fracasaba el tratamiento con FMSN o amoxicilina (por ineficacia) se procedieron a interrumpir el mismo e instaurar un tratamiento de segunda línea. La duración de los distintos periodos (tratamiento de primera línea y tratamiento de segunda línea) fue estimada de los datos del ensayo clínico y la Guía de Práctica Clínica Diagnóstico y Tratamiento de Sinusitis Aguda $^{18}$ y Adaptación de la Guía de Práctica Clínica ARIA 2010 para México. Metodología ADAPTE ${ }^{23}$. El tratamiento de FMSN en dicho estudio tuvo una duración media aproximada de 15 días y el tratamiento con amoxicilina 10 días $^{14}$, así como la duración del tratamiento de rescate de acuerdo a estas guías estuvo comprendido entre los 10 a 14 días.
El horizonte temporal del modelo es de 28 días, periodo en el que se incluye el tiempo de evaluación tratamiento de la RSA con ambos comparadores, así como el tiempo de rescate con una segunda línea en caso de falla del tratamiento ${ }^{14}$ en los pacientes para la evaluación de la eficacia y la tolerabilidad de FMSN y la amoxicilina administrados en el ensayo clínico.

Para la estimación del costo-efectividad incremental debido a la utilización de FMSN en el tratamiento RSA, se utilizó la siguiente fórmula:

$$
\Delta C / \Delta E=\sum_{i=1}^{n} \Delta C i / \sum_{i=1}^{n} \Delta E i
$$

Dónde:

$\Delta C$ : es la diferencia entre el costo de tratamiento con FMSN y la terapia con amoxicilina

$\Delta E$ : es la diferencia entre la eficacia de tratamiento con FMSN y la terapia con amoxicilina

De esta manera, se podrá estimar el costo incremental por paciente con mejoría en los síntomas con el uso de FMSN en comparación con amoxicilina.

El modelo se realizó en su totalidad el programa Excel en su versión 97-2003.

\section{RESULTADOS}

Los resultados básicos del análisis costo-efectividad de furoato mometasona se muestran en las tablas número 1,2 y 3 .

Tabla 1. Probabilidades utilizadas en el modelo del análisis costo-efectividad de furoato mometasona en el tratamiento de la RSA

Media D.E. Dist. Alfa Beta n

\section{Eficacias*}

Furoato Mometasona

Eficacia (\% de pacientes con mejoría de los síntomas)

$\%$ de pacientes que requieren consultas adicionales

$\%$ de pacientes que requieren tratamiento de 2 a línea

$\%$ de pacientes que requieren estudios de Laboratorio

$\%$ de petes sin mejoría con Radiodiagnóstico

Amoxicilina

Eficacia (\% de pacientes con mejoría de los síntomas)

$\%$ de pacientes que requieren consultas adicionales

$\%$ de pacientes que requieren tratamiento de 2 a línea

$\%$ de pacientes que requieren estudios de Laboratorio

$\%$ de petes sin mejoría con Radiodiagnóstico

Utilización de Recursos (Panel de expertos)**

Pacientes SIN mejoría

$\begin{array}{cccccc}0.67 & 0.03 & \text { Beta } & 162 & 81 & 243 \\ 0.32 & 0.03 & \text { Beta } & 77 & 166 & 243 \\ 0.32 & 0.03 & \text { Beta } & 77 & 166 & 243 \\ 0.32 & 0.03 & \text { Beta } & 77 & 166 & 243 \\ 0.32 & 0.03 & \text { Beta } & 77 & 166 & 243 \\ & & & & & \\ 0.61 & 0.03 & \text { Beta } & 154 & 97 & 251 \\ 0.37 & 0.03 & \text { Beta } & 92 & 159 & 251 \\ 0.37 & 0.03 & \text { Beta } & 92 & 159 & 251 \\ 0.37 & 0.03 & \text { Beta } & 92 & 159 & 251 \\ 0.37 & 0.03 & \text { Beta } & 92 & 159 & 251\end{array}$




\begin{tabular}{|c|c|c|c|c|c|c|}
\hline & Media & D.E. & Dist. & Alfa & Beta & n \\
\hline$\%$ de pacientes SIN mejoría que requiere consultas adicionales & 0.95 & 0.02 & Beta & 95 & 5 & 100 \\
\hline$\%$ de pacientes que requieren tratamiento de 2 a línea & 0.95 & 0.02 & Beta & 95 & 5 & 100 \\
\hline$\%$ de pacientes SIN mejoría que requiere laboratorio adicionales & 0.95 & 0.02 & Beta & 95 & 5 & 100 \\
\hline \% de pacientes SIN mejoría que requiere Radiodiagnóstico 1er nivel adicionales & 0.95 & 0.02 & Beta & 95 & 5 & 100 \\
\hline \multicolumn{7}{|l|}{ Pacientes con mejoría } \\
\hline Promedio de consultas en pacientes CON mejoría & 2 & 0.40 & Normal & & & \\
\hline Promedio de laboratorio en pacientes CON mejoría & 1 & 0.20 & Normal & & & \\
\hline Promedio de estudios de Radiodiagnóstico en pacientes SIN mejoría & 1 & 0.20 & Normal & & & \\
\hline Promedio de consultas en pacientes SIN mejoría & 4 & 0.80 & Normal & & & \\
\hline Promedio de laboratorio en pacientes SIN mejoría & 2 & 0.40 & Normal & & & \\
\hline Promedio de estudios de Radiodiagnóstico en pacientes SIN mejoría & 1 & 0.20 & Normal & & & \\
\hline Costo promedio del tratamiento de rescate & 185 & 57.37 & Gamma & 25 & 11 & \\
\hline
\end{tabular}

a) D.S. Desviación Estándar

b) Dis. Distribución

Fuentes: *(Meltzer EO et al.) - ** supuestos del modelo

En la Tabla 1, la eficacia se pudo expresar como la medida de la mejoría significativa de los síntomas asociados a RSA, según el IRC, durante el periodo de tratamiento analizando. Por su parte, en los puntajes de síntomas totales, basado en tres resultados que comúnmente se utilizan en los ensayos clínicos, se pudo observar, que el grupo de pacientes con FMSN $200 \mathrm{mcg}$ cada 12 horas tuvo un mejor resultado que el tratamiento con amoxicilina ( $67 \%$ vs. $61 \%$ ), así como que la eficacia de FMSN fue superior a la reportada por amoxicilina con un incremental de $5 \%$ con respecto a la otra opción.

Tabla 2. Estimación de la utilización y costos de los recursos sanitarios.

\begin{tabular}{|c|c|c|c|c|}
\hline Principio activo & Presentación & Posología & Costo Unitario & Costo/día \\
\hline \multicolumn{5}{|l|}{ Tratamiento * } \\
\hline Amoxicilina & Amoxicilina $500 \mathrm{mg}$, envase con 12 cap & $500 \mathrm{mg} \mathrm{3/dia/14días}$ & 2.04 & 28.58 \\
\hline $\begin{array}{l}\text { Furoato de } \\
\text { Mometasona }\end{array}$ & 140 nebulizaciones de $50 \mu \mathrm{g}$ cada una & $200 \mu \mathrm{g} \mathrm{c} / 12 \mathrm{~h} / 15$ días & 5.66 & 84.86 \\
\hline \multicolumn{5}{|c|}{ Tratamiento de rescate* } \\
\hline Clindamicina & Clindamicina $300 \mathrm{mg}$, envase con 16 cap & $300 \mathrm{mg}$ cada 6 horas & 2.53 & 35.35 \\
\hline TMP/SMX & $\begin{array}{c}\text { TMP/SMX (80/400mg envase con } 20 \\
\text { tabletas }\end{array}$ & $\begin{array}{l}\text { TMP/SMX160/800 } \\
\text { c12h/14 días }\end{array}$ & 0.87 & 12.15 \\
\hline Azitromicina & Azitromicina $500 \mathrm{mg}$ envase con 4 tab & $500 \mathrm{mg}$ por 3 días & 3.75 & 11.25 \\
\hline Doxiciclina & Doxiciclina $100 \mathrm{mg}$, envase con 10 cap & $100 \mathrm{mg} \mathrm{c} / 12 / 14$ dias & 4.02 & 56.28 \\
\hline Claritromicina & $\begin{array}{c}\text { Claritromicina } 250 \mathrm{mg} \text {, envase con } 10 \\
\text { cap }\end{array}$ & $500 \mathrm{mg} \mathrm{c} / 12 \mathrm{~h} / 14$ días & 7.16 & 100.24 \\
\hline \multicolumn{5}{|l|}{--Quinolonas } \\
\hline Levofloxacina & $500 \mathrm{mg}$ envase con $7 \mathrm{tab}$ & $500 \mathrm{mg} \mathrm{c} / 24$ h/10 días & 2.84 & 39.7 \\
\hline Moxifloxacina & $400 \mathrm{mg}$ envase con $7 \mathrm{tab}$ & $400 \mathrm{mg} \mathrm{c} / 24 \mathrm{~h} / 14$ días & 13.64 & 190.96 \\
\hline \multicolumn{5}{|l|}{-Cefalosporinas } \\
\hline Cefalotina & cefalotina $1 \mathrm{~g}$ amp $5 \mathrm{ml}$ & $1 \mathrm{~g} \mathrm{c} / 6 \mathrm{~h} 10$ días & 61.92 & 619.2 \\
\hline Cefuroxima & Cefuroxima $750 \mathrm{mg}$ amp $10 \mathrm{ml}$ & $750 \mathrm{mg} \mathrm{c} / 8 \mathrm{~h} 10$ días & 59.64 & 596.4 \\
\hline
\end{tabular}




\begin{tabular}{|c|c|c|c|}
\hline Principio activo & 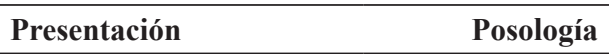 & Costo Unitario & Costo/día \\
\hline \multicolumn{4}{|c|}{ Estudios de gabinete y laboratorios $* *$} \\
\hline \multicolumn{4}{|c|}{--Primer nivel } \\
\hline Consulta & & 37.1 & \\
\hline Biometría hemática & & 5.4 & \\
\hline Rx de senos paranasales & & 12.2 & \\
\hline Rx lateral de cráneo & & 12.2 & \\
\hline \multicolumn{4}{|l|}{--Segundo nivel } \\
\hline Consulta & & 37.1 & \\
\hline Biometría hemática & & 5.4 & \\
\hline $\begin{array}{l}\text { Eosinofilos en Moco } \\
\text { nasal }\end{array}$ & & 9.6 & \\
\hline Rx de senos paranasales & & 16.7 & \\
\hline Rx lateral de cráneo & & 16.7 & \\
\hline
\end{tabular}

a) mg: miligramos

f) TMP/SMX: Trimetoprim sulfametoxazol

b) cap: capsulas

g) tab: tabletas

c) $\mu \mathrm{g}$ : microgramos

h) g: gramo

d) c: cada

i) ml: mililitro

e) h: horas

j) Rx: radiografía

Fuentes:

*IMSS. Portal de Compras IMSS. Disponible en: http://compras.imss.gob.mx/?P=imsscompro

** DOF/2016 Costos Unitarios de Atención Médica del IMSS. Jueves 25 de febrero de 2017. Disponible en: http://www.dof.gob.mx/nota detalle.php? codigo $=5427055 \&$ fecha $=25 / 02 / 2016$.

En la Tabla 2, se realizó una estimación de la utilización y los costos de los recursos sanitarios de los distintos

medicamentos y otros recursos sanitarios para tratar la RSA en el contexto del SNS de México.

Tabla 3. Resultados del Análisis de Costo efectividad de furoato mometasona en comparación con Amoxicilina en el tratamiento de la RSA en México.

\begin{tabular}{lccccc}
\hline Tratamiento RSA & Costo & Costo incremental & Eficacia & Eficacia incremental & RCEI \\
\hline Furoato de mometasona & $\$ 3,261$ & $\$(177)$ & 0.54 & 0.06 & \\
Amoxicilina- & $\$ 3,438$ & & 0.48 & & $\$(328)$ \\
\hline
\end{tabular}

a) RSA Rinosinusitis Aguda - b) RCEI Razón de costo efectividad Incremental

En la Tabla 3, se muestran los resultados del análisis de costo efectividad de furoato mometasona en comparación con amoxicilina en el tratamiento de la RSA en México. Los costos proyectados fueron de $\$ 3,261$ pesos con FMSN y $\$ 3,438$ pesos con amoxicilina. El FMSN fue asociado con un ahorro de costos por paciente de $\$ 177$ pesos en comparación con la amoxicilina en un periodo de dos semanas. La tasa incremental de costo-efectividad del FMSN lo coloca por encima de la amoxicilina. Como se puede observar en esta tabla, el FMSN $200 \mathrm{mcg}$ cada 12 horas ha demostrado ser una alternativa costo dominante, por ser más eficaz y tener un costo menor que la otra opción comparada.
Para comprobar la estabilidad de los resultados y la consistencia de las estimaciones efectuadas en el caso básico, se hizo un análisis de sensibilidad simple univariante. Con este análisis de sensibilidad, se identificaron las variables críticas que tienen un mayor impacto sobre los resultados finales.

En el diagrama de tornado, se resume el análisis de sensibilidad de los resultados del modelo a partir del RCEI. Este diagrama de tornado permite visualizar el impacto de la diversificación de las variables consideradas en un rango determinado en el resultado del modelo, Figura 2. 


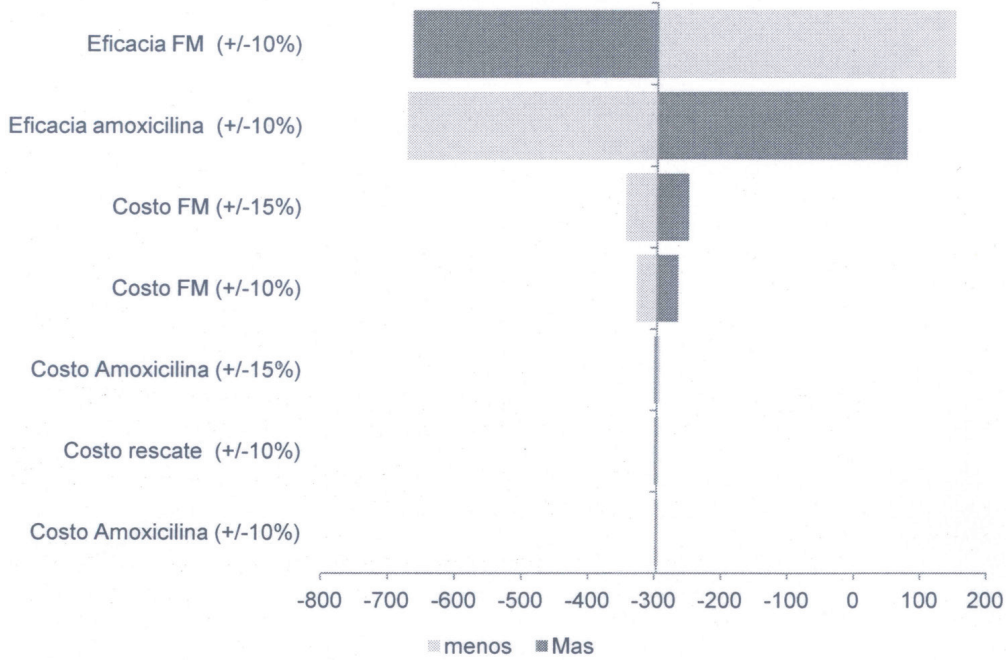

Figura 2. Diagrama de tornado: análisis de la sensibilidad y la relación costo efectividad incremental.

El análisis de sensibilidad univariado realizado muestra que la variable que más influyó en el resultado del modelo fue la eficacia del tratamiento del FMSN. Además, en todos los casos, tanto al aumentar como disminuir el precio de FMSN en $10 \%$ y $15 \%$, esta alternativa farmacológica sigue siendo una opción costo-efectiva, al ser la opción dominante, porque los cambios en los precios de amoxicilina de $( \pm 10 \%)$ tienen poco impacto en los resultados. Por lo tanto, en todas las variaciones se observa que FMSM presenta un ahorro para el SNS, por lo que se puede considerar que los resultados del modelo son robustos.

El plano de costo-efectividad muestra que la mayor parte de los puntos representados se concentran en el cuadrante de menor costo y mayor efectividad. Se puede apreciar que el tratamiento con FMSN 200 mcg cada 12 horas, también resulta ser una terapia ahorradora para el SNS mexicano, Figura 3.

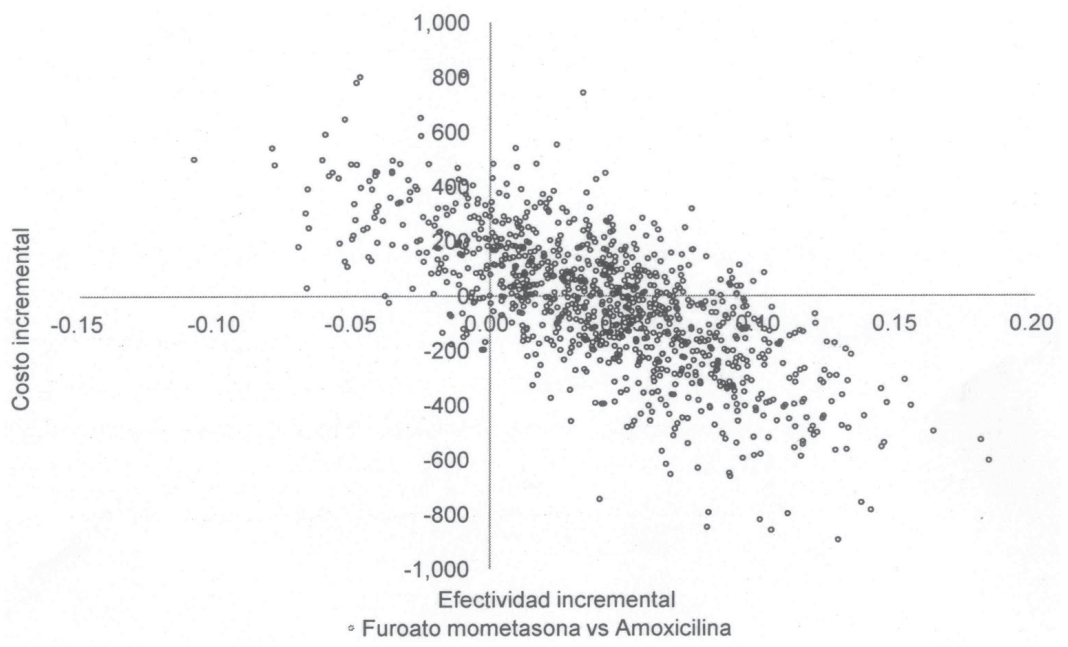

Figura 3. Plano de costo efectividad de Furoato mometasona vs. Amoxicilina en el tratamiento de los síntomas RSA.

\section{DISCUSIÓN}

La RSA es un problema sanitario que conlleva una elevada carga financiera para el SNS de México. Los antibióticos están perdiendo eficacia por el aumento progresivo de la resistencia bacteriana, lo que es una preocupación para la salud pública en el país ${ }^{24-26}$. El incremento de la resistencia a los antibióticos deriva de su uso inapropiado, especialmente en situaciones que pueden resolverse con otras clases terapéuticas, como en el caso de RSA ${ }^{27}$. 
El incremento en la utilización de los antibióticos, su mal uso y otros factores relacionados, han dado lugar a la emergencia de cepas resistentes ${ }^{28-29}$. La Organización Mundial de la Salud (OMS) ${ }^{30}$ ha promovido el uso racional de medicamentos ${ }^{31}$ y recomendado que sea integrado en políticas nacionales ${ }^{32}$. En México, se ha implementado una política para restringir la venta y uso inadecuado de antibióticos ${ }^{33}$, pero todavía es insuficiente la información publicada sobre la situación actual o que describa la respuesta que se ha dado a esta situación, así como la evidencia primordial para formular y evaluar políticas $^{34-35}$.

El presente estudio muestra que las diferencias en el costo de adquisición de FMSN para el tratamiento de la RSA, se compensan por su efectividad diferencial, ya que el costo medio por paciente de las dos opciones fue $\$ 3,261$ y $\$ 3,438$ para FMSN y amoxicilina, respectivamente. Los resultados respecto a la mejoría de los síntomas, mostraron que el FMSN se asocia a una mayor eficacia en relación con la amoxicilina, con ganancias de $5 \%$ de mejoría. Los resultados coinciden con publicaciones recientes, por lo que son consistentes y confiables ${ }^{36}$.

El presente estudio tiene algunas limitaciones importantes que deben tenerse en cuenta. En primer lugar, la eficacia se estimó a partir de dos ensayos clínicos comparativo disponible. Por otra parte, la utilización de recursos se estimó parcialmente a partir de guías de actualización clínica, y de la opinión de un panel de expertos, debido a la no disponibilidad de algunos datos en el ensayo o a la necesidad de simular la actividad de la práctica clínica en México.

Otra limitación a destacar de este trabajo, es la no consideración de los costos de los efectos adversos de los tratamientos. Aunque su inclusión no cambiaría de forma relevante el sentido de los resultados obtenidos, dada la mayor incidencia de toxicidad de los antibióticos respecto a FMSN, la creciente preocupación por la seguridad asociada a los antibióticos en los últimos años, además de los nuevos datos sobre resistencia de los antibióticos, hace que los resultados del presente análisis justifiquen justifica el uso de FMSN en el tratamiento de la RSA.

Otra posible limitación de este estudio, es el hecho de que únicamente se han tenido en cuenta los costos directos del tratamiento. No obstante, evitar eventos como la resistencia bacteriana asociada mediante la utilización de amoxicilina reportará unos beneficios en términos de evitación de pérdidas de productividad por absentismo, baja laboral e incapacidad temporal o permanente que no han sido estudiados en el presente trabajo. En cualquier caso, parece claro que la inclusión de este tipo de costos favorecería la utilización de FMSN, al contabilizar los ahorros en términos de pérdidas de productividad evitadas por la resistencia bacteriana.

Cabe destacar algunas fortalezas del modelo, ya que en la mayoría de las estimaciones de utilización de los recursos, se hizo a partir de los resultados de los ensayos clínico aleatorizado, doble ciego, y no mediante estimaciones teóricas. Además, se hicieron varios análisis de sensibilidad que confirman la estabilidad de los resultados del caso básico. Finalmente, los aspectos clínicos del modelo fueron validados por un panel de expertos clínicos en México.

\section{CONCLUSIONES}

Se pudo demostrar que el FMSN a 200 mcg cada 12 horas comparado con la amoxicilina $500 \mathrm{mg}$ cada ocho horas, en primera línea de tratamiento en la RSA, es una terapia costo-efectiva dominante y que adicionalmente pudiera ser una terapia ahorradora de hasta $\$ 90$ pesos por paciente, debido principalmente a su mayor eficacia, lo cual implica menos gastos en tratamiento en caso de falla terapéutica, estudios de laboratorio, gabinete y hospitalización.

El análisis de sensibilidad univariado realizado muestra que las variables que más influyó en el resultado del modelo resulto ser la eficacia, no obstante, el costo unitario de FMSN con una variación de entre 10\% y $15 \%$ sigue situándose dentro del intervalo de eficiencia. Por lo tanto, al tener en cuenta los datos del estudio y los resultados del análisis de sensibilidad, el estudio puede considerarse lo suficientemente robusto en sus conclusiones, como para afirmar que la terapia con FMSN 200 mcg cada 12 horas, es un tratamiento costoahorrador y costo-efectivo para el SNS mexicano.

\section{AGRADECIMIENTOS}

Al Laboratorio Merck Sharp \& Dohme de México por la subvención realizada.

\section{CONSIDERACIONES ÉTICAS}

El presente trabajo no tiene problemas de índole ético ya que es modelaje que no utiliza información de fuentes primarias (información personal de los pacientes), y solo retoma la información de efectividad clínica corresponde a estudios previamente publicados. 


\section{CONFLICTO DE INTERESES}

Los autores del presente trabajo no tienen ningún conflicto de interés, a pesar de que el estudio fue patrocinado con una subvención sin restricciones de Merck Sharp \& Dohme de México.

\section{REFERENCIAS}

1. Piccirillo JF. Acute bacterial sinusitis. N Engl J Med. 2004. DOI:351:902-910. 10.1056/NEJMcp035553

2. Hickner JM, Bartlett JG, Besser RE, Gonzales R, Hoffman JR, Sande MA. Principles of appropriate antibiotic use for acute rhinosinusitis in adults: background. Ann Intern Med. 2001; 134(6): 498505. DOI: 10.7326/0003-4819-134-6-20010320000017.

3. Secretaría de Salud. Dirección General de Epidemiología. Anuarios de Morbilidad 2015.

4. Secretaría de Salud. Observatorio de los Servicios de Atención Primaria 2012. Dirección General de Evaluación del Desempeño. Secretaría de Salud. México, 2013.

5. Ray NF, Baraniuk JN, Thamer M, Rinehart CS, Gergen PJ, Kaliner S. et al. Healthcare expenditures for sinusitis in 1996: contributions of asthma, rhinitis, and other airway disorders. J Allergy Clin Immunol. 1999; 103(3): 408-414. DOI: doi. org/10.1016/S0091-6749(99)70464-1.

6. Benninger MS, Holzer SES, Lau J. Diagnosis and treatment of uncomplicated acute bacterial rhinosinusitis: summary of the Agency for Health Care Policy and Research evidence-based report. Otolaryngology-Head and Neck Surgery. 2000; 122(1): $1-7$.

7. Scadding GK. Other anti-inflammatory uses of intranasal corticosteroids in upper respiratory inflammatory diseases.Allergy. 2000; 55(s62): 19-23. DOI: 10.1034/j.1398-9995.2000.055suppl62019.x.

8. Meltzer EO, Hamilos DL, Hadley JA, DC Lanza, Marple BF, Nicklas RN et al. Rhinosinusitis: establishing definitions for clinical research and patient care. J Allergy Clin Immunol. 2004; 114(6): 155-212. DOI: http://doi.org/10.1016/j. jaci.2004.09.029.

9. Lund VJ. Therapeutic targets in rhinosinusitis: infection or inflammation? The Medscape J Med. 2008; 10(4): 105.

10. Angier E, Willington J, Scadding G, Holmes S, Walker S, Management of allergic and non-allergic rhinitis: a primary care summary of the BSACI guideline. Prim Care Respir J. 2010; 19(3): 217-222.

11. Thomas M, Yawn BP, Price D, Lund V, Mullol
J, Fokkens W, et al. EPOS Primary guidelines: European Position Paper on the primary care diagnosis and management of rhinosinusitis and nasal polyps 2007. Prim Care Respir J. 2008; 17(2): 79-89. DOI: $10.3132 /$ pcrj.2008.00029.

12. Penagos M, Compalati E, Tarantini F, BaenaCagnani CE, Passalacqua G, Canonica GW. Efficacy of mometasone furoate nasal spray in the treatment of allergic rhinitis. Meta-analysis of randomized, double-blind, placebo-controlled, clinical trials. Allergy. 2008; 63(10): 1280-1291. DOI: 10.1111/j.1398-9995.2008.01808.x.

13. Canonica WG, Compalati E, Tarantini F, Passalacqua G, Penagos M. Efficacy of mometasone furoate nasal spray in the treatment of allergic rhinitis: metaanalysis of randomized, double-blind, placebocontrolled clinical trials. J Allergy Clin Immunol. 2008; 121(2): S52.

14. Meltzer EO, Bachert C, Staudinger H. Treating acute rhinosinusitis: comparing efficacy and safety of mometasone furoate nasal spray, amoxicillin, and placebo. J Allergy Clin Immunol. 2005; 116(6): 1289-1295.

15. Rosenfeld R, Andes D, Bhattacharyya N, Cheung D, Eisenberg S, et al. Clinical practice guideline: adult sinusitis. Otolaryngology, Head and Neck Surgery 2007. 137, S1-131.

16. Rosenfeld R, Piccirillo J, Chandrasekhar S, Brook I, Kumar AK, Kramper M. et al. Clinical practice guideline (update): adult sinusitis. Otolaryngol Head Neck Surg. 2015; 152, (2S) S1-39. DOI: $10.1177 / 0194599815572097$.

17. Svensson J, Lundberb J, Olsson P, Stjárne P, Tennvall G. Cost effectiveness of mometasone furoate nasal spray in the treatment of acute rhinosinusitis. Prim Care Respir J. 2012; 21(4): 412-418. DOI: 10.4104/ pcrj.2012.00085.

18. Guía de práctica clínica diagnóstico y tratamiento de la rinosinusitis aguda en la edad pediátrica, México: Secretaría de Salud, 2010.

19. Chow AW, Benninger MS, Brook I, Brozek JL, Goldstein EJ, Hicks LA, et al. IDSA clinical practice guideline for acute bacterial rhinosinusitis in children and adults. Clin Infect Dis. 2012; 54(8): e72-e112. DOI: $10.1093 / \mathrm{cid} / \mathrm{cir} 1043$.

20. Meltzer EO, Gates D, Bachert C. Mometasone furoate nasal spray increases the number of minimalsymptom days in patients with acute rhinosinusitis. Ann Allergy, Asthma Immunol. 2012; 108(4): 275279. DOI: 10.1016/j.anai.2012.01.015.

21. Guiscafré-Gallardo H. La atención integral del niño con infección respiratoria aguda: lo apropiado y lo actual. Guías clínicas para disminuir el abuso 
de antimicrobianos y de sintomáticos, así como el de evitar que los niños mueran en el hogar por neumonía, después de haber recibido consulta médica. Bol Med Hosp Infant Mex. 2008; 65(4): 249-260.

22. Consejo de Salubridad General. Guía para la conducción de estudios de evaluación económica para la actualización del Cuadro Básico de Insumos del Sector Salud en México. Dirección General Adjunta de Priorización. Comisión interinstitucional del Cuadro Básico de Insumos del Sector Salud. 2008.

23. Lareas-Linnemann D, Mayorga-Butrón JL, Sánchez-González AS, Ramírez -García A, MedinaÁvalos M, Figueroa-Morales MA, et al. ARIA México 2014. Adaptación de la Guía de Práctica Clínica ARIA 2010 para México. Metodología ADAPTE. Rev Alergia Mex. 2014; 61(S1): 3-116.

24. Rodríguez Moreno C, Campoamor Landín F, Zaforteza Dezcallar M, Verdejo González A, Muro Pascual V, Martín Martín MV. Política de antibióticos en atención primaria: la experiencia práctica en un área sanitaria. Aten Primaria. 1998; 21(5): 315-320.

25. Smith RD, Coast J. Antimicrobial resistance: a global response. Bulletin of the World Health Organization. 2002; 80(2): 126-133.

26. Kumar A. Microbial resistance to drugs-a universal problem in urgent need of a comprehensive approach. Nat Med J India. 1997; 10(5): 221-224.

27. Barbosa TM, Levy SB. The impact of antibiotic use on resistance development and persistence. Drug Resist Updat. 2000; 3(5): 303-311. DOI: 10.1054/ drup.2000.0167.

28. Nathwani D, Malek M. Cost considerations in the evaluation of new therapies for gram-positive bacteria. Int J Antimicrob Agents. 1999;13(2):71-78.

29. Chrischilles EA, Scholz DA. Dollars and sense: a practical guide to cost analysis for hospital epidemiology and infection control. Clin Perform Qual Health Care. 1999; 7(2): 107-111.

30. Giske CG, Monnet DL, Cars O, Carmeli Y. Clinical and economic impact of common multidrugresistant gram-negative bacilli. Antimicrob Agents Chemother. 2008; 52(3): 813-21. DOI: 10.1128/ AAC.01169-07.

31. World Health O. The Rational Use of Drugs. Report of the Conference of Experts 1985. Geneva: WHO; 1987. Either first page or author must be supplied.

32. World Health O. How to develop and implement a national drug policy: World Health Organization; 2001.

33. Origlieri C, Bielory L. Intranasal corticosteroids and allergic rhinoconjunctivitis. CurrenT Opin Allergy Clin Immunol. 2008; 8(5): 450-456. DOI: $10.1097 /$ ACI.0b013e32830d8501.

34. World Health O. Comparative analysis of national drug policies in 12 countries; 1996.

35. World Health O. WHO policy perspectives on medicines: promoting rational use of medicines: core components. Geneva: WHO; 2002 Sep: WHO/ EDM;2002.

36. Lange B, Lukat KF, Rettig K, Holtappels G, Bachert C. Efficacy, cost-effectiveness, and tolerability of mometasone furoate, levocabastine, and disodium cromoglycate nasal sprays in the treatment of seasonal allergic rhinitis. Ann Allergy, Asthma Immunol. 2005; 95(3): 272-282. DOI: 10.1016/ S1081-1206(10)61225-2.

\section{COMENTARIO EDITORIAL}

En este número publicamos un estudio de evaluación económica de un esteroide para el tratamiento de adultos con rinosinusitis aguda ${ }^{1}$. Dado el interés creciente en este tipo de estudios para aprobar la inclusión de tecnología sanitaria dentro de los planes de servicios, su lectura e interpretación de los hallazgos debe hacerse con rigor $^{2}$. Estos trabajos suelen verse con cierto tipo de precaución dado que en buen número suelen ser patrocinados por empresas farmacéuticas, e incluso llegan a configurarse como elementos del mercadeo. Por ello, algunas recomendaciones para el lector de estos trabajos:

- Los resultados tienen una perspectiva, ya sea institucional, hospitalaria, social, etcétera, que debe ser considerada en la interpretación. No es posible extrapolar una perspectiva a otra perspectiva de análisis, porque los hallazgos podrían ser incluso contrarios. En este caso la perspectiva fue desde el sistema de salud mexicano, y no implica resultados desde el punto de vista de los pacientes o de la sociedad en general.

- Los hallazgos no deben ser extrapolados a contextos diferentes del cual fue hecho el estudio. Si bien la eficacia o efectividad de una intervención, bajo ciertos supuestos, podría ser generalizable, los contextos del sistema de salud y político son diferentes e imposibilita la extrapolación. En este caso el sistema de salud mexicano tiene especificidades diferentes a los de otros países, como pueden ser los de América Latina.

- Cuando se usan datos de otros estudios (casi siempre ensayos clínicos), como ocurrió en este caso, es importante que el lector conozca si hubo 
o no conflicto de interés en ese otro estudio. En el ensayo clínico original hubo un autor trabajador del laboratorio farmacéutico productor del medicamento.

- Cuando se usan datos de ensayos clínicos que evalúan eficacia de una tecnología en salud, los resultados no deben entenderse como que así funcionaría en la cotidianidad clínica. Si bien suele usarse el término "efectividad" al incorporar los datos en un estudio de evaluación económica, es más preciso hablar de costo-eficacia, para indicar que esto ocurriría sólo bajo las condiciones más óptimas.

- Debido a que los estudios de los que se extraen los datos usados en las evaluaciones económicas no suelen reportar los efectos adversos, el tema de seguridad de la tecnología en salud no suele ser incluido. En ese sentido, este tipo de estudios suelen verse sesgados, al igual que los ensayos clínicos, a solo mostrar los beneficios de la tecnología.

Como se puede apreciar, las evaluaciones económicas pueden tener problemas de interpretación como cualquier otro tipo de estudio, pese a ser rigurosos en relación a los métodos usados. Es importante que los profesionales interesados en estos temas se capaciten en el análisis crítico de estos estudios, cada vez más importantes para la toma de decisiones basadas en la evidencia.

Alvaro J. Idrovo

Editor Salud UIS

\section{REFERENCIAS}

1. Rely K, Salinas G, Martínez S, Pérez C, Blandón V, Alexandre PK. Costo-efectividad de furoato de mometasona tópica nasal para el tratamiento de la rinosinusitis aguda en México. Rev Univ Ind Santander. Salud 2017; 49(2): 312-321. DOI: http:// dx.doi.org/10.18273/revsal.v49n2-2017004.

2. Hill SR, Mitchell AS, Henry DA. Problems with the interpretation of pharmacoeconomic analyses: a review of submissions to the Australian Pharmaceutical Benefits Scheme. JAMA. 2000; 283(16): 2116-2121. DOI:10.1001/ jama.283.16.2116. 